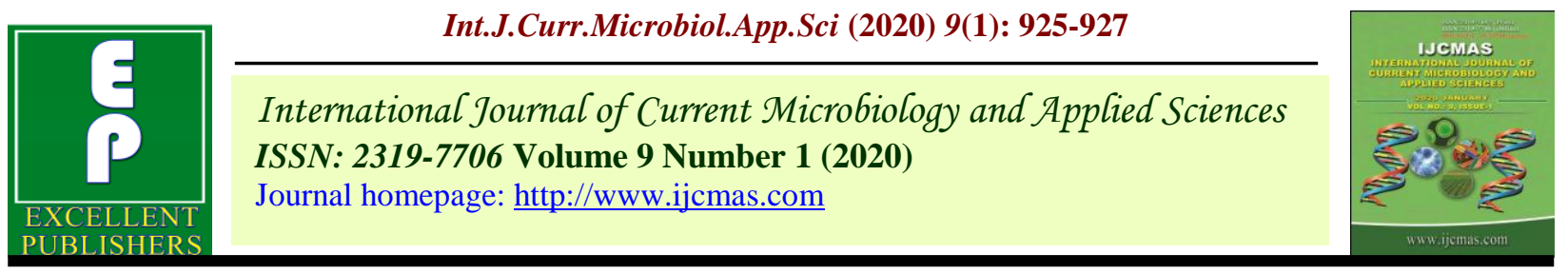

Original Research Article

https://doi.org/10.20546/ijcmas.2020.901.103

\title{
Impact of Varied Groups of Organics at Different Levels Supplied to Mulberry on Pupal and Egg Traits of Silkworm
}

\author{
A. Ravikumar ${ }^{1}$, B. V. Shreenivas ${ }^{2 *}$ and Raghavendra $^{3}$ \\ ${ }^{1}$ Agricultural Research Station, Hagari, Ballari, UAS, Raichur, India \\ ${ }^{2}$ Soil Science, Krishi Vigyan Kendra Kalaburagi, UAS, Raichur, India \\ ${ }^{3}$ Soil Science, UAS, Raichur, India \\ *Corresponding author
}

\section{Keywords}

Mulberry, Organics, Silkworm, Pupal and egg traits

Article Info

Accepted:

15 December 2019

Available Online:

20 January 2020

\section{A B S T R A C T}

The experiment was conducted in the Agriculture Research Station, Hagari, University of Agricultural Sciences Raichur under irrigated condition during 2017-18 to study the application of different groups of organics to mulberry along with urea viz., FYM+ urea $(75 \%+25 \%, 50 \%+50 \%$ and $25 \%+75 \%)$, Green leaf manure + urea $(75 \%+25 \%, 50 \%+50 \%$ and $25 \%+75 \%)$ and Castor cake + urea $(75 \%+25 \%, 50 \%+50 \%$ and $25 \%$ $+75 \%$ ), the effects of feeding such leaves to silkworm on the resultant pupal and egg traits was investigated. Worms fed on mulberry leaves obtained by application of Castor cake + urea recorded higher pupal weight (1.34 g), shorter pupal duration (11.38 days), higher rate of moth emergence (93.89\%), fecundity (495.94 eggs /laying) and hatchability $(95.03 \%)$ as compared to control (1.34g, 11.94 days, 91.99\%, 470.99 eggs and $93.93 \%$, respectively).

\section{Introduction}

The total area under mulberry in Karnataka is about 1,04,577.66 hectares of which about 11,592 MT of raw silk is produced per year (Anon. 2018). The growth and development of silkworms and the quality of produced are greatly affected by the quality of mulberry leaves. The nutritive value of mulberry leaf is important in improving and enhancing the cocoon quality. Though lot of information is available on the use of various organic manures and their positive effect on agricultural crops limited work has been done on the impact of varied groups of organics at different levels supplied to mulberry and their effect on pupal and egg traits in silkworm.

\section{Materials and Methods}

Study was carried out during 2017-18 in the established $V_{1}$ mulberry garden under 
irrigated condition. Organic manures such as FYM, Green leaf manure (GLM) and Castor cake (CC) at three levels (to supply 75\%, 50\% and $25 \% \mathrm{~N}$ ), FYM + GLM + CC + Urea (all to supply $25 \% \mathrm{~N})$, Urea $(100 \% \mathrm{~N})$ and recommended NPK as control were maintained. Mulberry leaves obtained with these treatments were fed to silkworm as per the standard rearing practices (Dandin et al., 2003) and the influence of same on pupal and egg traits was recorded. The data were analyzed statistically (Contrast for group mean) as outlined by Cochran and Cox (2000).

\section{Results and Discussion}

Data on the influence of different sources of nitrogen to mulberry through organics and their effect on grain age traits in mulberry silkworm are furnished in Table 1.

Table.1 Influence of various groups of organics applied to mulberry at different levels on grainage traits of Bombay mori $\mathrm{L}$.

\begin{tabular}{|c|c|c|c|c|c|c|c|c|c|c|}
\hline \multirow[t]{2}{*}{$\begin{array}{l}\text { Treat- } \\
\text { ments }\end{array}$} & \multicolumn{2}{|c|}{$\begin{array}{l}\text { Pupal weight } \\
\text { (g) }\end{array}$} & \multicolumn{2}{|c|}{$\begin{array}{c}\text { Pupal duration } \\
\text { (days) }\end{array}$} & \multicolumn{2}{|c|}{$\begin{array}{c}\text { Moth emergence } \\
(\%)\end{array}$} & \multicolumn{2}{|c|}{$\begin{array}{l}\text { Fecundity } \\
\text { (eggs/laying) }\end{array}$} & \multicolumn{2}{|c|}{ Hatching (\%) } \\
\hline & $\begin{array}{c}\text { Treatment } \\
\text { mean }\end{array}$ & $\begin{array}{c}\text { Group } \\
\text { mean }\end{array}$ & $\begin{array}{c}\text { Treatment } \\
\text { mean }\end{array}$ & $\begin{array}{l}\text { Group } \\
\text { mean }\end{array}$ & Treatment mean & $\begin{array}{l}\text { Group } \\
\text { mean }\end{array}$ & $\begin{array}{c}\text { Treatment } \\
\text { mean }\end{array}$ & $\begin{array}{l}\text { Group } \\
\text { mean }\end{array}$ & $\begin{array}{l}\text { Treatment } \\
\text { mean }\end{array}$ & $\begin{array}{l}\text { Group } \\
\text { mean }\end{array}$ \\
\hline $\mathbf{T}_{1}$ & $\begin{array}{l}1.30 \\
1.35 \\
1.32\end{array}$ & $1.32^{\mathrm{NS}}$ & $\begin{array}{l}12.34 \\
10.96 \\
11.76\end{array}$ & $11.68 *$ & $\begin{array}{l}90.26(71.87) \\
94.71(76.74) \\
92.50(74.13)\end{array}$ & $11.68^{*}$ & $\begin{array}{l}445.00 \\
527.83 \\
470.33\end{array}$ & $481.00^{*}$ & $\begin{array}{l}93.08(74.83) \\
95.40(77.82) \\
94.12(76.09)\end{array}$ & $481.00^{*}$ \\
\hline $\mathbf{T}_{2}$ & $\begin{array}{l}1.31 \\
1.36 \\
1.34\end{array}$ & $1.33^{\mathrm{NS}}$ & $\begin{array}{l}12.23 \\
10.96 \\
11.59\end{array}$ & $11.59 *$ & $\begin{array}{l}90.94(72.53) \\
95.99(78.51) \\
93.02(74.74)\end{array}$ & $11.59 *$ & $\begin{array}{l}444.50 \\
529.50 \\
485.66\end{array}$ & $486.55^{*}$ & $\begin{array}{l}93.45(75.27) \\
96.18(78.88) \\
94.30(76.33)\end{array}$ & $486.55^{*}$ \\
\hline $\mathbf{T}_{3}$ & $\begin{array}{l}1.31 \\
1.38 \\
1.34\end{array}$ & $1.33 *$ & $\begin{array}{l}12.08 \\
10.64 \\
11.42\end{array}$ & 11.38 & $\begin{array}{l}91.77(73.39) \\
96.66(79.60) \\
93.24(74.98)\end{array}$ & $11.38^{*}$ & $\begin{array}{l}455.50 \\
540.33 \\
492.00\end{array}$ & $495.94 *$ & $\begin{array}{l}93.79(75.70) \\
96.76(79.83) \\
94.56(76.65)\end{array}$ & $495.94 *$ \\
\hline Controls & $\begin{array}{l}1.36 \\
1.30 \\
1.35\end{array}$ & 1.34 & $\begin{array}{l}11.95 \\
12.69 \\
11.18\end{array}$ & $11.94 *$ & $\begin{array}{l}92.24(73.85) \\
89.87(71.51) \\
93.88(75.76)\end{array}$ & $11.94 *$ & $\begin{array}{l}464.00 \\
437.33 \\
511.66\end{array}$ & $470.99^{*}$ & $\begin{array}{l}93.95(74.83) \\
92.85(74.57) \\
94.99(77.24)\end{array}$ & $470.99 *$ \\
\hline $\begin{array}{l}\text { S.Em } \\
\pm\end{array}$ & \multicolumn{2}{|c|}{$\begin{array}{c}0.018 \\
-\end{array}$} & \multicolumn{2}{|c|}{$\begin{array}{l}0.129 \\
0.365\end{array}$} & \multicolumn{2}{|l|}{$\begin{array}{c}0.616 \\
1.744\end{array}$} & \multicolumn{2}{|c|}{$\begin{array}{c}24.51 \\
69.34\end{array}$} & \multicolumn{2}{|c|}{$\begin{array}{l}0.876 \\
2.478\end{array}$} \\
\hline $\begin{array}{l}\text { C.D.at } \\
5 \%\end{array}$ & & & & & & & & & & \\
\hline
\end{tabular}

*Significant (Group means): Values in the parentheses are angular transformed.

\begin{tabular}{|c|c|c|c|}
\hline $\mathrm{T}_{1}=75 \% \mathrm{~N}-\mathrm{FYM}+25 \% \mathrm{~N}-$ & $\mathrm{T}_{2}=75 \% \mathrm{~N}-\mathrm{GLM}+25 \% \mathrm{~N}-$ & $\mathrm{T}_{3}=75 \% \mathrm{~N}-\mathrm{CC}+25 \% \mathrm{~N}-$ & Controls \\
Urea & Urea & Urea & $25 \% \mathrm{~N}-\mathrm{FYM}+25 \% \mathrm{~N}-\mathrm{GLM}$ \\
$50 \% \mathrm{~N}-\mathrm{FYM}+50 \% \mathrm{~N}-$ Urea & $50 \% \mathrm{~N}-\mathrm{GLM}+75 \% \mathrm{~N}-$ Urea & $50 \% \mathrm{~N}-\mathrm{CC}+50 \% \mathrm{~N}-$ Urea & $+25 \% \mathrm{NC}+25 \% \mathrm{~N}-$ Urea \\
$25 \% \mathrm{~N}-\mathrm{FYM}+75 \% \mathrm{~N}-$ Urea & $25 \% \mathrm{~N}-\mathrm{GLM}+75 \% \mathrm{~N}-$ Urea & $25 \% \mathrm{~N}-\mathrm{CC}+75 \% \mathrm{~N}-$ Urea & $100 \%$ Urea \\
& & & Recommended NPK + FYM \\
\hline
\end{tabular}




\section{Pupal duration}

Significantly higher pupal weight was recorded in respect of worms fed on mulberry leaves obtained by application of 50\% N through CC $+50 \%$ through Urea $(1.38 \mathrm{~g})$. Among different organic manures tried, CC group yielded higher pupal weight $(1.34 \mathrm{~g})$ which was found to be on par with control group (1.34g) followed by GLM (1.33g) and FYM group (1.32g).

\section{Rate of moth emergence}

Significantly higher rate of moth emergence was noticed in case of CC + Urea $(93.89 \%)$ followed by GLM + Urea (93.31\%) and FYM + Urea $(92.49 \%)$.

\section{Fecundity}

Fecundity was higher in moths emerged from pupae formed worms which were fed on mulberry leaves obtained by application of $\mathrm{CC}+$ Urea at different levels (495.94 eggs/laying) and the next best were GLM + Urea (486.55 eggs/laying) and FYM + Urea (481 eggs/laying) compared to control (470.99 eggs/laying).

\section{Hatching percentage}

In different organic groups, significantly higher hatching percentage was recorded in the treatment of CC + Urea at different levels (95.03\%), followed by GLM + Urea (94.64\%) and FYM + Urea $(94.40 \%)$. Hatching was lower in case of control $(9.093 \%)$. These results are inconformity with those of Shankar et al., (1992) who reported that the use of organic manures in six splits even at $10 \mathrm{t} \mathrm{ha}^{-1}$ year $^{-1}$ with $50 \%$ inorganic fertilizer had no deleterious effect on grainage parameters. The application of FYM + recommended NPK recorded highest grainage parameters followed by sheep manure + recommended NPK (Rajanna et al., 2000).

\section{References}

Anonymous, 2018, Annual report. Dept. of Sericulture, Government of Karnataka. Pp. 250-253.

Cochran, W.C. and Cox, G. M,2000, Experimental Designs, $2^{\text {nd }}$ Edn., Oxford and IBH Pub., New York.

Dandin, S.B., Jayant Jayaswal and Giridhar, K. 2003, Handbook of Sericulture Technologies. CSB, Bangalore, p.284.

Rajanna, B.H, Chinnaswamy, K.P., Govindan, R., Sannappa, B and Sundar Raj, S, 2000, Influence of Sericulture byproducts and other organic manures on rearing performance of $\mathrm{NB}_{4} \mathrm{D}_{2}$ silkworm (Bombyx mori L.) breed. In: Silkworm Management in Tropics (Eds. R. Govindan, K.P. Chinnaswamy, N.K. Krishna Prasad and D.N.R. Reddy). Prod. Natl. Sem. Tropic. Seric., UAS, Bangalore, 2:192-194.

Shankar, M.A., Shivashankar, K. and Devaiah, M.C. 1992, Influence of organic manure and fertilizer levels on cocoon yield, silk yield and quality. Mysore J. Agric. Sci., 26:180-187.

\section{How to cite this article:}

Ravikumar, A., B. V. Shreenivas and Raghavendra. 2020. Impact of Varied Groups of Organics at Different Levels Supplied to Mulberry on Pupal and Egg Traits of Silkworm. Int.J.Curr.Microbiol.App.Sci. 9(01): 925-927. doi: https://doi.org/10.20546/ijcmas.2020.901.103 